\title{
Anaesthetic Management of a Spontaneously Ruptured Vertebral Artery Pseudoaneurysm with Dynamic Airway Obstruction
}

\author{
Abeywardana $C \mathrm{Y}^{1^{*}}$, Fernando $\mathrm{M}^{2}$, Silva $\mathrm{H} \mathrm{A} \mathrm{S}^{3}$ \\ ${ }^{1}$ Senior Registrar in Anesthesiology, ${ }^{2}$ Senior Consultant Anesthetist, ${ }^{3}$ Registrar in Anesthesiology, \\ National Hospital of Sri Lanka, Colombo, Sri Lanka
}

\begin{abstract}
Expanding neck hematoma with progressive airway obstruction is a life-threatening airway emergency. Patients are at risk of cranio-cerebral circulation compromise leading to perioperative cerebrovascular accidents. Furthermore, precipitous fall of blood pressure following neck hematoma exploration mandate the activation of protocols for massive transfusion. We describe the successful anaesthetic management of an extremely rare case of spontaneous rupture of a vertebral artery pseudoaneurysm.
\end{abstract}

Keywords: airway obstruction; vertebral artery pseudoaneurysm rupture; difficult airway; massive transfusion

\section{Introduction}

Expanding neck hematoma with progressive airway obstruction in an unprepared patient presents unique challenges to the anaesthetist in terms of emergency airway control and perioperative hemodynamic stabilization. This case report describes the challenges faced by the anaesthetist following spontaneous rupture of a vertebral artery pseudoaneurysm.

\section{Case report}

A 29-year old man, under investigation for pyrexia of unknown origin (PUO), was diagnosed with a pseudoaneurysm arising from the $3^{\text {rd }}$ segment of the left vertebral artery. He did not have any focal neurological deficits. His cardiac, biochemical and hematological investigations were normal except for progressive drop of haemoglobin for which one unit of blood was transfused.

Prior to scheduled radiological intervention of pseudoaneurysm, patient experienced sudden

$$
\begin{aligned}
& \text { *Correspondence: Abeywardana CY } \\
& \text { E mail: charaka3@yahoo.co.uk }
\end{aligned}
$$

https://orcid.org/0000-0002-1708-4509

Received: $12 / 09 / 2020$

Accepted: $29 / 10 / 2020$

DOI: http:/doi.org/10.4038/slja.v29i1.8681 worsening of neck pain soon after dinner followed by rapid enlargement of neck swelling, dysphagia, drooling of saliva, hoarseness of voice and stridor. His symptoms worsened in supine position. He was anxious with a blood pressure of $157 / 95 \mathrm{mmHg}$ and a pulse rate of $117 /$ minute. $\mathrm{SpO}_{2}$ was $96 \%$ in room air. Respiratory rate was 22/minute. A tender, tense progressively enlarging swelling was present on the left side of neck with extension to the upper back and the nape of the neck. The neck was flexed with a right sided tilt with complete restriction of extension. Mouth opening was about $2 \mathrm{~cm}$. Tongue protrusion was intact. Mallampati grading could not be assessed. Landmarks for front of neck access were obscure. ABG revealed $\mathrm{pH}$ of 7.44, $\mathrm{P}_{\mathrm{a}} \mathrm{CO}_{2}$ of $35 \mathrm{mmHg}, \mathrm{P}_{\mathrm{a}} \mathrm{O}_{2}$ of $132 \mathrm{mmHg}$ (with $6 \mathrm{~L} /$ min Oxygen via face mask), $\mathrm{HCO}_{3}$ of 24.8 $\mathrm{mmol} / \mathrm{L}$ and lactate of $3.5 \mathrm{mmol} / \mathrm{L}$. Clinical examination was conclusive for rupture of left vertebral artery pseudoaneurysm with rapid neck hematoma formation and progressive airway obstruction. Emergency neck exploration and vascular repair was planned.

Multi-disciplinary team (MDT) input was taken with involvement of the anaesthetist, vascular surgeon, ENT surgeon and transfusion physician. Immediate risk to life and risks of surgery were explained to the patient. Massive transfusion protocol (MTP) was activated. Difficult airway management equipment was brought to the theatre. IV Ranitidine $50 \mathrm{mg}$ bolus was administered. Stepwise airway plan was drawn up, accepting the risks according to the Difficult 
Airway Society (DAS) guidelines. First option was awake fibre-optic nasal intubation (AFONI). Failing which, inhalational induction or emergency front-of neck surgical airway access (FONSA) were considered.

Monitoring was established according to AAGBI standard. Patient was oxygenated with nasal prongs at $4 \mathrm{~L} /$ minute before and during intubation with head-end elevated. Airway was topicalized with nebulized $100 \mathrm{mg} 1 \%$ lignocaine. AFONI was performed by the most experienced endoscopist via right nostril. It revealed swollen soft tissues of the nasopharynx and supra-glottis. The subglottic airway was normal. $6.5 \mathrm{~mm}$ reinforced nasal endotracheal tube was inserted. Co-induction was performed with propofol, midazolam, fentanyl and atracurium. Right femoral central venous and right radial arterial catheters were inserted. Anaesthesia was maintained with $\mathrm{O}_{2}$, air and isoflurane. Surgical exploration revealed profound arterial bleeding from friable artery fragments from where the pseudoaneurysm was located with extensive soft tissue oedema. Haemostasis was achieved with packing of the surgical field and diathermy. Left internal and external jugular veins were ligated. Morphine was administered in titrated doses. Intraoperative hemodynamic fluctuations were managed with judicious use of noradrenaline and blood products. Intraoperatively SBP was maintained above $100 \mathrm{mmHg}$, mean arterial pressure (MAP) above $75 \mathrm{mmHg}$ and a urine output of more than $0.3 \mathrm{ml} / \mathrm{kg} /$ hour. Intraoperative blood loss was $2530 \mathrm{cc}$. Patient received $5 \mathrm{U}$ red cell concentrates, $4 \mathrm{U}$ FFP and $1500 \mathrm{cc}$ of crystalloids. IV Tranexamic acid, calcium gluconate and meropenem bolus were administered. Point-of-care (POC) coagulation test performed at end of surgery was normal. Patient was hemodynamically stable without vasopressor support at end of surgery. $\mathrm{ABG}$ at end of surgery revealed a $\mathrm{pH}$ of 7.4, $\mathrm{P}_{\mathrm{a}} \mathrm{CO}_{2}$ of 47 $\mathrm{mmHg}, \mathrm{P}_{\mathrm{a}} \mathrm{O}_{2}$ of $291 \mathrm{mmHg}, \mathrm{HCO}_{3}$ of $28 \mathrm{mmol} / \mathrm{L}$ with resolving lactate of $2.9 \mathrm{mmol} / \mathrm{L}$. He was electively paralyzed and ventilated for 48 hours.

\section{Figure 1}

Appearance following rupture of pseudoaneurysm

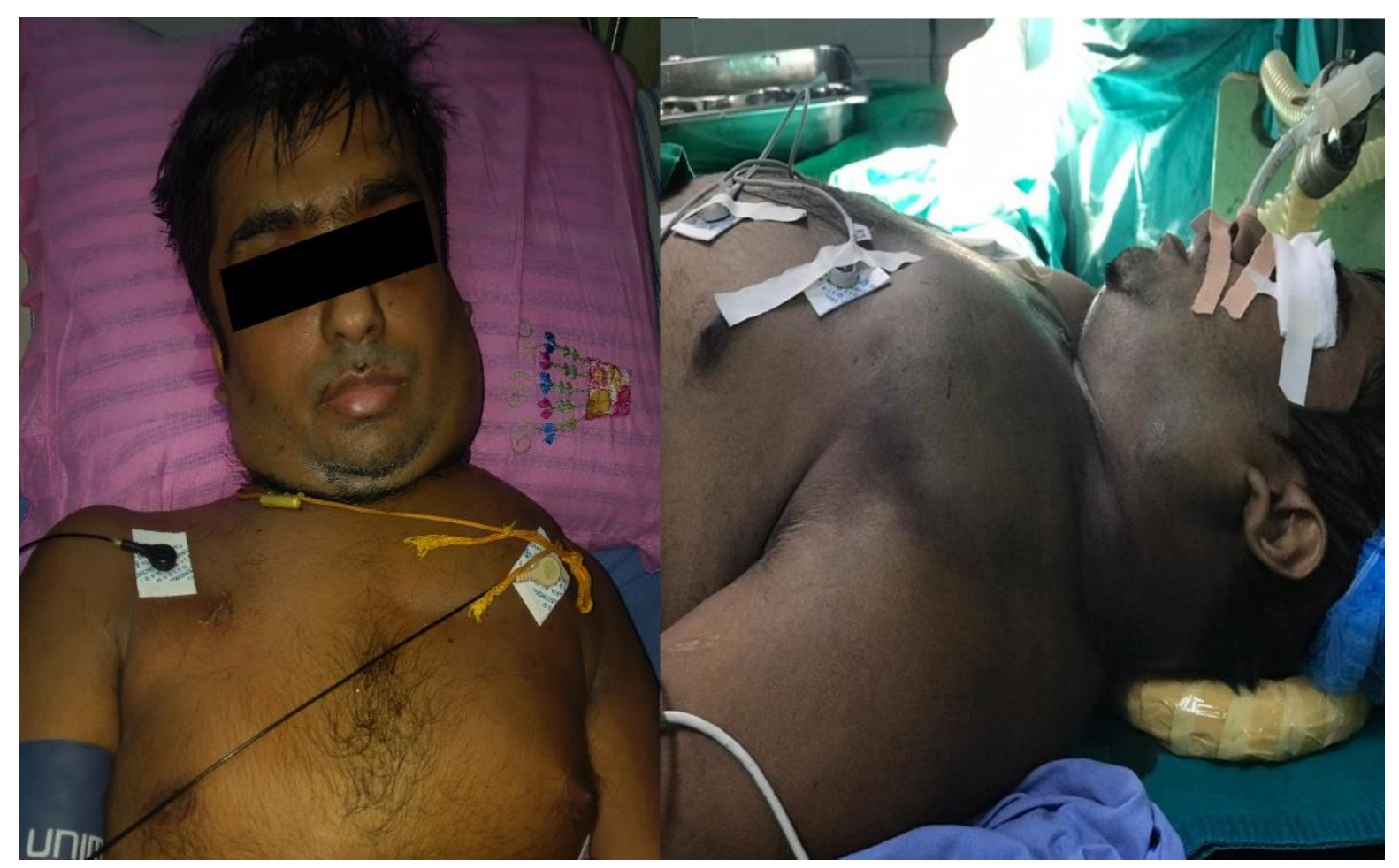




\section{Figure 2}

Postoperative appearance at day 28

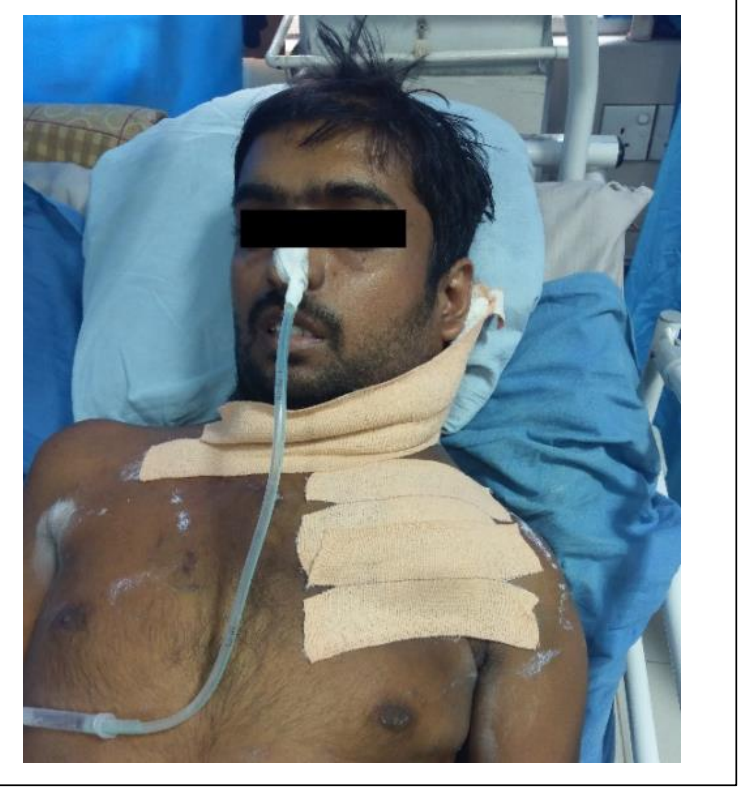

CT brain performed on $2^{\text {nd }}$ postoperative day was normal. Digital subtraction angiography excluded retrograde filling of the affected arterial system. An incidental finding of right internal carotid artery fusiform dilatation was noted. Packs were removed on $4^{\text {th }}$ postoperative day under anaesthesia. Patient was extubated on $5^{\text {th }}$ postoperative day. He was discharged home on $34^{\text {th }}$ day of illness with no residual neurological deficit.

\section{Discussion}

Vertebral artery pseudoaneurysms commonly form following blunt neck trauma and head injuries. ${ }^{1}$ Majority of vertebral artery pseudoaneurysms remain clinically silent. ${ }^{1,2}$ Symptoms and signs are mainly neurological. Onset of clinical features depend on the patency of the collateral circulation ${ }^{2}$. There was no history of cervical trauma in this patient. Deep fascia of the neck resists soft tissue expansion following haemorrhage. Haematoma result in venous congestion which impair venous drainage from the head and neck region which receive about 12$15 \%$ of resting cardiac output. ${ }^{3,4}$ This leads to soft tissue oedema and progressive airway obstruction ${ }^{4}$. Venous bleeding presents with gradual airway obstruction whereas arterial bleeding into soft tissues of neck lead to rapid obstruction as in this patient. ${ }^{4}$ Haematoma can extend to chest, back and opposite side of neck along fascial planes thus affecting the uninjured regions worsening airway obstruction as exhibited in this patient. Therefore, urgent control of bleeding is paramount to prevent progressive airway obstruction. ${ }^{5,6}$

Anticipated perioperative anaesthetic issues were; difficult airway and intubation, risk of aspiration, limited IV access, possible massive transfusion, anxious uncooperative patient, perioperative cerebrovascular event risk and postoperative airway management. Airway was approached stepwise according to DAS guidelines. ${ }^{5,6}$ AFONI was selected as the initial method because of availability of experts and clinical airway examination was favorable. Ability to protrude tongue denoted relative absence of edema of floor-of-mouth. LMA was not considered as backup airway plan due to restricted mouth opening. FONSA access was considered as the rescue method as neck landmarks were not visible and the potential risk of catastrophic bleeding upon neck deep fascia release. DAS recommend preoperative neck ultrasound in anticipated difficult airway management to mark the midline. It was not performed in this patient due to the emergency nature of the surgery and difficulty in visualization. ${ }^{5,6}$

Lower limb IV access were considered to prevent exacerbation of neck venous congestion. Surgical manipulation, carotid sinus stimulation by expanding hematoma and haemorrhage can result in perioperative bradycardia and hypotension ${ }^{7}$. Hence an arterial line is essential to monitor and correct rapid hemodynamic fluctuations. Collateral circulation of the brain should be maintained to prevent intraoperative cerebral ischemia. ${ }^{3,7}$ Therefore, SBP and MAP targets were maintained thoroughly with activation of MTP and judicious vasopressor use. POC tests are useful for early detection and correction of coagulopathy.

Postoperative airway plan should be established in advance. This patient was paralyzed and ventilated to prevent re-bleeding due to coughing and bucking. This facilitated resolution of neck oedema. Extubation should be considered only once re-bleeding is negated radiologically and surgically in addition to fulfillment of standard extubation criteria.

History of PUO and subsequent detection of another fusiform dilatation of right carotid artery 
in this patient probably points towards a vasculitic aetiology for pseudoaneurysm formation. Further investigation regarding aetiology is warranted.

\section{Conclusion}

Progressive airway obstruction following rupture of vertebral artery pseudoaneurysm is extremely rare. Early MDT intervention and a stepwise airway plan according to DAS guidelines aids in decision making and rational patient management. Consideration of cerebrovascular physiology and establishment of hemodynamic targets should be instituted prior to surgery to prevent cerebral ischemia. Rational approach to postoperative airway management is important to prevent re-bleeding and postoperative airway obstruction.

\section{Consent}

Direct consent was obtained from the patient for this publication and use of photographs.

\section{Declaration of interest}

None.

\section{References}

1. Schittek A. Pseudoaneurysm of the vertebral artery. Tex Heart Inst J. 1999; 26(1): 90-5. PMID: 10217474
2. Daou B, Hammer C, Chalouhi N. Dissecting pseudoaneurysms: predictors of symptom occurrence, enlargement, clinical outcome, and treatment. J Neurosurg. 2016 Oct; 125(4): 936-42. https://doi.org/10.3171/2015.10.JNS151846 PMID: 26824374

3. Tameem A, Krovvidi H. Cerebral physiology. Contin Educ Anaesth Crit Care Pain. 2013 Aug;13(4): 113-8.

https://doi.org/10.1093/bjaceaccp/mkt001

4. Lee HS, Lee BJ, Kim SW. Patterns of Postthyroidectomy Hemorrhage. Clin Exp Oto rhino laryngol. 2009; 2(2): 72. https://doi.org/10.3342/ceo.2009.2.2.72 PMID: 19565031

5. Price TM, McCoy EP. Emergency front of neck access in airway management. BJA Educ. 2019 Aug; 19(8): 246-53. https://doi.org/10.1016/j.bjae.2019.04.002

6. Ahmad I, El-Boghdadly K, Bhagrath R. Difficult Airway Society guidelines for awake tracheal intubation (ATI) in adults. Anaesthesia [Internet]. 2019 Nov 14 [cited 2020 Jan 30] http://doi.wiley.com/10.1111/anae.14904

7. Stoneham MD, Thompson JP. Arterial pressure management and carotid endarterectomy. $\mathrm{Br} J$ Anaesth. 2009 Apr; 102(4): 442-52. https://doi.org/10.1093/bja/aep012 